\title{
COMPLETION OF A CONSTRUCTION OF JOHNSTONE
}

\author{
JOHN ISBELL
}

\begin{abstract}
A complete lattice is constructed which is not sober in the Scott
\end{abstract} topology.

Peter Johnstone has constructed [3] a (countable) partially ordered set $X^{+}$which admits a sober topology (i.e. the relation $x \in\{y\}^{-}$gives the partial order) but is not sober in the Scott topology. He asked whether such an example can be a complete lattice; this note shows that it can.

Some of the background should be mentioned, as it is in [3]. Every complete lattice admits a sober topology; but the topology that R.-E. Hoffmann used for this [2] is a bit tricky to deal with. There is a condition for sobriety of the Scott topology on a complete lattice $[1, \Pi, 4.14]$ which Johnstone calls "mild".

The definition of the Scott topology of a partially ordered set is just this: a set is closed provided it contains all predecessors of its elements and suprema of its nonempty up-directed subsets.

The following construction is rather closely based on Johnstone's; but, to make it work in a complete lattice, I need uncountably many coatoms. Let $\omega$ be the set of natural numbers, $\omega+1=\omega \cup\{\omega\}$, and $A$ the set of $\left(2^{\omega}\right)$ ordinals less than $2^{\omega}$. Let $B=\omega \times(\omega+1), C=\omega^{\omega} \times \omega$. Order $B$ by $(m, n) \leq\left(m^{\prime}, n^{\prime}\right)$ if $(1)$ $m=m^{\prime}$ and $n \leq n^{\prime}$, or (2) $n^{\prime}=\omega$ and $m<m^{\prime}$. Order $C$ by $(f, n) \leq\left(f^{\prime}, n^{\prime}\right)$ if $f=f^{\prime}$ and $n \leq n^{\prime}$. Let $I \subset A^{2} \times B \times C$ be the set of all ordered quadruples $(\alpha, \beta,(m, n),(f, \bar{k}))$ for which $\alpha \neq \beta, n=f(m)$ (thus $n \neq \omega)$, and $k=m$. The power of $I$ is only $2^{\omega}$, so there is an injection $i: I \rightarrow A$. We can require, too, that $i(\alpha, \beta,(m, f(m)),(f, m))>\alpha \vee \beta$; and we call it $i(\alpha, \beta, f, m)$ for short.

Let $S$ consist of $B \cup C$ with a greatest element $T$ added (elements of $B$ being incomparable with elements of $C$ ). Let $X=A \times S$. Generate a partial order on $X$ (by transitive closure) from (1) the order of $S$ in each fiber $\{\alpha\} \times S$, and (2) the relations $(i(\alpha, \beta, f, m), T) \geq(\alpha,(m, f(m)))$ and $(i(\alpha, \beta, f, m), T) \geq(\beta,(f, m))$.

Observe that up-directed subsets of $X$ have suprema. Our complete lattice $L$ consists of the intersections of principal order ideals in $X$. Most of what we have to check is that the nonprincipal ideals in $L$ are "sparse" and do not affect the up-directed sets. First, the principal ideals are the sequences $\{(\alpha,(m, x)): x \leq n\}$, with $n$ finite or $m=0$, the unions of $m+1$ sequences $\{(\alpha,(x, y)): x \leq m\}$, the finite sequences $\{(\alpha,(f, z)): z \leq n\}$, the sets $\{\alpha\} \times S$ with $\alpha$ not a value of $i$, and one more type: for $\gamma=i(\alpha, \beta, f, m)$, the predecessors of $(\gamma, T)$ form $\{\gamma\} \times S$, a finite sequence under $(\alpha,(m, f(m)))$, and a finite sequence under $(\beta,(f, m))$. The intersections include of course $\emptyset$ and $X$. We shall find the remaining ones, the nontrivial intersections. Observe that an intersection contained in one fiber $\{\alpha\} \times S$

Received by the editors October 22, 1981.

1980 Mathematics Subject Classification. Primary 06B30; Secondary 54F05.

Key words and phrases. Scott topology, sober, complete lattice.

(C) 1982 American Mathematical Society 0002-9939/81/0000-0394/\$01.50 
is trivial. So we need to look at intersections of (first) pairs of principal ideals of the last type, under $(\gamma, T)=(i(\alpha, \beta, f, m), T)$ and $\left(\gamma^{\prime}, T\right)=\left(i\left(\alpha^{\prime}, \beta^{\prime}, f^{\prime}, m^{\prime}\right), T\right)$. If the intersection $M$ is nontrivial then $\{\alpha, \beta, \gamma\}$ and $\left\{\alpha^{\prime}, \beta^{\prime}, \gamma^{\prime}\right\}$ have two common elements-not three, since the largest elements $\gamma, \gamma^{\prime}$ are different. Impossible that $\alpha=\alpha^{\prime}, \beta=\beta^{\prime}$ (and $M$ nontrivial). For if $(m, f(m))$ and $\left(m^{\prime}, f^{\prime}\left(m^{\prime}\right)\right)$ have a common predecessor we have $m=m^{\prime}$; since $\gamma \neq \gamma^{\prime}, f \neq f^{\prime}$ and $(f, m)$ has no common predecessor with $\left(f^{\prime}, m\right)$. Impossible, of course, that $\alpha=\beta^{\prime}$ and $\beta=\alpha^{\prime}$. Choosing notation so that $\gamma^{\prime}>\gamma$, the possible cases are (1) $\gamma=\alpha^{\prime}, \beta=\beta^{\prime}$, and (2) $\gamma=\beta^{\prime}, \alpha=\alpha^{\prime}$. In case (1), a nontrivial intersection $M$ must contain $(\beta,(f, 0))$, so $f^{\prime}=f$. No third principal ideal $J$ has nontrivial intersection with $M$. It could only be under $\left(\gamma^{\prime \prime}, T\right)=\left(i\left(\alpha^{\prime \prime}, \beta^{\prime \prime}, f^{\prime \prime}, m^{\prime \prime}\right), T\right) ; \gamma^{\prime \prime} \neq \beta$, since then $J$ would not meet $\{\gamma\} \times S$, so $\beta^{\prime \prime}=\beta$ and $\alpha^{\prime \prime}=\gamma$. Again $f^{\prime \prime}=f$; so $m^{\prime \prime} \neq m^{\prime}$ (since $\gamma^{\prime \prime} \neq \gamma^{\prime}$ ), and $\left(m^{\prime \prime}, f\left(m^{\prime \prime}\right)\right)$ has no common predecessor with $\left(m^{\prime}, f\left(m^{\prime}\right)\right)$, making the intersection trivial. It follows that two nontrivial intersections are incomparable if one of them is in this case.

Similarly, in case (2), common predecessors of $(\alpha,(m, f(m)))$ and $\left(\alpha,\left(m^{\prime}, f^{\prime}\left(m^{\prime}\right)\right)\right)$ require $m^{\prime}=m$. A third principal ideal under $\left(\gamma^{\prime \prime}, T\right)$ having nontrivial intersection with $M$ requires $\alpha^{\prime \prime}=\alpha=\alpha^{\prime}, \beta^{\prime \prime}=\beta^{\prime}, m^{\prime \prime}=m^{\prime}$; so $f^{\prime \prime} \neq f^{\prime}$ and the parts in $\{\gamma\} \times S$ are disjoint. And all nontrivial intersections are incomparable. Also, each has only finitely many predecessors. So embedding $X$ in $L$ by principal ideals preserves suprema of up-directed sets.

Now in the complete lattice $L$, the complement $L^{-}$of the singleton $\{X\}$ certainly is not the Scott closure of a point. But $L^{-}$is Scott closed: obviously downward closed, and an up-directed subset has at most one nontrivial element and has its supremum in $L^{-}$. Finally, $L^{-}$has no disjoint nonempty open sets $U_{1}, U_{2}$. They would contain maximal elements $\left(\alpha_{1}, T\right)$ resp. $\left(\alpha_{2}, T\right)$. Then $U_{1}$ must contain almost all of the elements $\left(\alpha_{1},(n, \omega)\right)$ (all for $n \geq n_{1}$ ); and for each of those $n$ it must contain all $\left(\alpha_{1},(n, x)\right)$ for $x \geq f(n)$, for some $f \in \omega^{\omega}$. Now if $U_{1}$ and $U_{2}$ are disjoint, $\alpha_{1} \neq \alpha_{2}$; and $U_{2}$ contains almost all $\left(\alpha_{2},(f, y)\right)$, all for $y \geq n_{2}$. Pick $n \geq n_{1} \vee n_{2}$, and we have $\left(\alpha_{1},(n, f(n))\right) \in U_{1},\left(\alpha_{2},(f, n)\right) \in U_{2}$. So both contain $\left(i\left(\alpha_{1}, \alpha_{2}, f, n\right), T\right)$, a contradiction.

\section{REFERENCES}

1. G. Gierz, K. H. Hofmann, K. Keimel, J. D. Lawson, M. Mislove and D. S. Scott, A compendium of continuous lattices, Springer-Verlag, Berlin and New York, 1980.

2. R.-E. Hoffmann, Essentially complete $T_{0}$-spaces, Manuscripta Math. 27 (1979), 401-431.

3. P. T. Johnstone, Scott is not always sober, Lecture Notes in Math., vol. 871, Springer-Verlag, Berlin and New York, 1981, pp. 282-283.

Department of Mathematics, State University of New York, Buffalo, New YORK 14214 